\title{
A Novel Design of Sparse Prototype Filter for Nearly Perfect Reconstruction Cosine-Modulated Filter Banks
}

\author{
Wei Xu ${ }^{1,2}$, Yi Li ${ }^{1}\left(\mathbb{D}\right.$, Jinghong Miao ${ }^{1, *}$, Jiaxiang Zhao ${ }^{3}$ and Xin Gao ${ }^{1}$ \\ 1 School of Electronics and Information Engineering, Tianjin Polytechnic University, Tianjin 300387, China; \\ doctorxw@126.com (W.X.); piaopiaojiazu@outlook.com (Y.L.); gaoxin_621@163.com (X.G.) \\ 2 Tianjin Key Laboratory of Optoelectronic Detection Technology and System, Tianjin 300387, China \\ 3 College of Electronic Information and Optical Engineering, Nankai University, Tianjin 300071, China; \\ zhaojx@nankai.edu.cn \\ * Correspondence: miaojinghong@tjpu.edu.cn; Tel.: +86-022-83955524
}

Received: 15 April 2018; Accepted: 21 May 2018; Published: 22 May 2018

\begin{abstract}
Cosine-modulated filter banks play a major role in digital signal processing. Sparse FIR filter banks have lower implementation complexity than full filter banks, while keeping a good performance level. This paper presents a fast design paradigm for sparse nearly perfect-reconstruction (NPR) cosine-modulated filter banks. First, an approximation function is introduced to reduce the non-convex quadratically constrained optimization problem to a linearly constrained optimization problem. Then, the desired sparse linear phase FIR prototype filter is derived through the orthogonal matching pursuit (OMP) performed under the weighted $l_{2}$ norm. The simulation results demonstrate that the proposed scheme is an effective paradigm to design sparse NPR cosine-modulated filter banks.
\end{abstract}

Keywords: cosine-modulated; filter banks; FIR; sparse

\section{Introduction}

Maximally decimated, uniform, cosine-modulated filter banks play a major role in applications where signals must be processed in sub-bands [1-5]. There are two main methods obtaining a cosine-modulated filter bank. (1) Design analysis and synthesis filter banks respectively under the certain conditions; (2) Design one or two prototype filters and get the analysis and synthesis filter banks by cosine modulating. In this paper, we choose the later one because the design is straightforward, e.g., this method focus on designing a single prototype filter.

There are several methods to design the prototype filter of cosine-modulated filter banks [6-8]. The weighted constrained least square (WCLS) algorithm proposed in reference [6] casts the design problem as a linear minimization of prototype filter coefficients, which makes the value at $\omega=\pi / 2 M$ is 0.707 . However, the order of the prototype filter is very high. It is generally true that group delay increases with the order of the filter. The quantum-behaved particle swarm optimization (QBPSO) algorithm proposed in reference [7] appropriately relaxes the limitation of perfect-reconstruction conditions. This method designs the prototype filter by making an unconstrained optimization to adjust the pass band cutoff frequency of objective function, which minimizes the cost function to meet the reconstruction conditions. Although the amplitude distortion is guaranteed, the aliasing distortion cannot meet the perfect-reconstruction condition of filter banks, and the attenuation of prototype filter is restricted. The design method based on gradient information (GI) in reference [8] uses the gradient vector of objective function to design prototype filter. However, the order of prototype coefficient is high, the aliasing distortion and the amplitude distortion of filter banks are not controlled well. 
The order of NPR cosine-modulated filter bank designed by these methods is high and not sparse, which makes an increasing number of the multipliers and adders in practical application, and results in a high hardware cost and power consumption.

In this paper, we present a fast design paradigm for sparse NPR cosine-modulated filter banks. A sparse FIR filter bank contains a large number of zero coefficients, such that the multipliers and adders corresponding to these zero coefficients are not required, which results in a lower hardware cost and power consumption with allowed accuracy of distortion. The design procedure can be divided into two stages. In the first stage, an approximation function $f(\omega)$ is introduced to reduce the non-convex quadratically constrained optimization problem to a linearly constrained optimization problem. The desired sparse linear phase FIR prototype filter can be derived through the orthogonal matching pursuit [9] performed under the weighted $l_{2}$ norm in the second stage. The simulation results demonstrate that the proposed scheme is an effective paradigm to design sparse NPR cosine-modulated filter banks.

The paper organization is as follows: In Section 2, we briefly summarize the design framework of perfect-reconstruction cosine-modulated filter banks. The design of sparse linear phase FIR prototype filter of NPR cosine-modulated filter banks is showed in Section 3. The representative experimental results and comparison data are discussed in Section 4. The conclusion of proposed scheme is showed in Section 5.

\section{Perfect-Reconstruction Cosine-Modulated Filter Banks}

A $M$-channel maximally decimated filter bank as illustrated in Figure 1 is composed of $M$ finite impulse response duration analysis filters with impulse responses $h_{k}(n)$, the down- and up- sampling by factor $M$ of sub-band signals, and synthesis FIR filters $g_{k}(n)$. The impulse response of filter bank is obtained by cosine modulation with a low pass filter:

$$
\begin{aligned}
& h_{k}(n)=2 h(n) \cos \left[\frac{\pi}{M}\left(k+\frac{1}{2}\right)\left(n-\frac{V}{2}\right)+(-1)^{k} \frac{\pi}{M}\right], \\
& g_{k}(n)=2 h(n) \cos \left[\frac{\pi}{M}\left(k+\frac{1}{2}\right)\left(n-\frac{V}{2}\right)-(-1)^{k} \frac{\pi}{M}\right]
\end{aligned}
$$

with $0 \leq k \leq M-1$ and $0 \leq n \leq N-1 . h(n)$ is the impulse response of the FIR prototype filter, $V$ denotes the system delay and $N$ is the order of the prototype filter. The input-output relation of the system in the $z$ domain is given by

$$
\hat{X}(z)=A_{0}(z) X(z)+\sum_{l=1}^{M-1} A_{l}(z) X\left(z e^{-j 2 \pi l / M}\right)
$$

where

$$
A_{0}(z)=\frac{1}{M} \sum_{k=0}^{M-1} H_{k}(z) G_{k}(z)
$$

is the distortion transfer function which determining the distortion of non-aliased component $X(z)$ caused by the system, and

$$
A_{l}(z)=\frac{1}{M} \sum_{k=0}^{M-1} H_{k}\left(z e^{-j 2 \pi l / M}\right) G_{k}(z)
$$

are the aliasing transfer functions which determine the attenuation of aliased components $X\left(z e^{-j 2 \pi l / M}\right)$ for $l=1, \ldots, M-1$. It follows that the filter bank holds the perfect-reconstruction property if and only if 


$$
\begin{aligned}
& A_{0}(z)=z^{-V} \\
& A_{l}(z)=0, \quad l=1, \ldots, M-1 .
\end{aligned}
$$

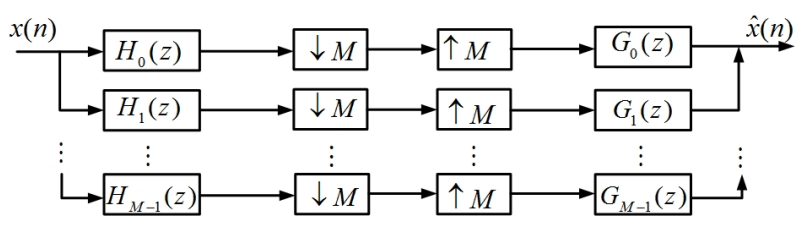

Figure 1. M-channel maximally decimated filter bank.

\section{The Design of Sparse Linear Phase FIR Prototype Filter of NPR Cosine-Modulated Filter Banks}

Without loss of generality, the linear phase FIR filter $H\left(e^{j \omega}\right)$ is assumed to be the Type $I I$ which suggests that the length $N$ of $H\left(e^{j \omega}\right)$ is even and its tap weights satisfying $h(n)=h(N-1-n)$ for all $0 \leq n \leq N-1$. For the other types of the linear phase filters, an argument similar to the one developed in this paper can be followed. The frequency response corresponding to an $\mathrm{N}$-th order Type II linear phase FIR prototype filter can be represented as

$$
\begin{aligned}
H\left(e^{j \omega}\right) & =e^{-j \frac{N-1}{2} \omega} H_{0}(\omega) \\
& =e^{-j \frac{N-1}{2} \omega}\left[2 \sum_{n=0}^{\frac{N}{2}-1} h(n) \cos \left(\left(\frac{N-1}{2}-n\right) \omega\right)\right] .
\end{aligned}
$$

where $H_{0}(\omega)$ is the real-valued amplitude response of prototype filter. The perfect-reconstruction conditions Equations (6) and (7) for linear phase cosine-modulated filter banks can be reduced to

$$
\begin{aligned}
\left|H_{0}\left(e^{j \omega}\right)\right|^{2}+\left|H_{0}\left(e^{j\left(\omega-\frac{\pi}{M}\right)}\right)\right|^{2} & =1, \omega \in\left[0, \frac{\pi}{M}\right], \\
\left|H_{0}\left(e^{j \omega}\right)\right| & =0, \omega \in\left(\frac{\pi}{M}, \pi\right] .
\end{aligned}
$$

Thus, the problem of sparse linear phase FIR prototype filter design for cosine-modulated filter banks can be formulated as

$$
\begin{array}{r}
\min \|\mathbf{h}\|_{0} \\
\text { s.t. } \quad|\mathbf{c}(\omega) \mathbf{h}|^{2}+\left|\mathbf{c}\left(\omega-\frac{\pi}{M}\right) \mathbf{h}\right|^{2}=1, \omega \in\left[0, \frac{\pi}{M}\right], \\
|\mathbf{c}(\omega) \mathbf{h}|=0, \omega \in\left(\frac{\pi}{M}, \pi\right]
\end{array}
$$

where we have

$$
\begin{aligned}
\mathbf{c}(\omega) & =\left[\cos \frac{N-1}{2} \omega, \cdots, \cos \left(\frac{N-1}{2}-n\right) \omega, \cdots, \cos \frac{1}{2} \omega\right], \\
\mathbf{h} & =2\left[h(0), h(1), \cdots, h(n), \cdots, h\left(\frac{N}{2}-1\right)\right]^{\mathrm{T}} .
\end{aligned}
$$

Define a approximation function as $f(\omega)$, which is required to satisfy the following equations:

$$
\begin{gathered}
f\left(\omega_{0}\right)=1, \\
f^{2}(\omega)+f^{2}\left(\omega-\frac{\pi}{M}\right)=1, \omega \in\left(\omega_{0}, \frac{\pi}{M}-\omega_{0}\right), \\
f\left(\frac{\pi}{M}-\omega_{0}\right)=0
\end{gathered}
$$


where $\omega_{0}=\alpha \pi / 2 M$ and $0 \leq \alpha \leq 1$. There are lots of methods to obtain the function $f(\omega)$. In this paper, we establish a mathematical model like

$$
f(\omega)=\cos (\kappa \omega-\phi) .
$$

Substituting Equation (14a), Equation (14c) and $f(\pi / 2 M)=\sqrt{2} / 2$ into Equation (15), we can obtain

$$
\kappa=\frac{M}{2(1-\alpha)}, \quad \phi=\frac{M \omega_{0}}{2(1-\alpha)} .
$$

Utilizing the approximation function $f(\omega)$, the problem of designing sparse NPR cosine-modulated filter banks can be expressed as a constrained optimization problem for the coefficients of prototype filter in the following form:

$$
\begin{gathered}
\min \quad\|\mathbf{h}\|_{0} \\
\text { s.t. }|\mathbf{c}(\omega) \mathbf{h}-1| \leq \delta_{p}, \omega \in\left[0, \omega_{0}\right), \\
|\mathbf{c}(\omega) \mathbf{h}-f(\omega)| \leq \delta_{t}, \omega \in\left[\omega_{0}, \frac{\pi}{M}-\omega_{0}\right], \\
|\mathbf{c}(\omega) \mathbf{h}| \leq \delta_{s}, \omega \in\left(\frac{\pi}{M}-\omega_{0}, \pi\right], \\
\mathbf{c}\left(\frac{\pi}{2 M}\right) \mathbf{h}=\frac{\sqrt{2}}{2}
\end{gathered}
$$

where $\delta_{p} \delta_{t}$ and $\delta_{s}$ represent the ripples of pass band, transition band and stop band respectively. To compute a solution of problem Equation (17), we follow the standard discretized procedure and replace the continuous parameter $\omega$ with discretized samples distributed in the frequency set $[0, \pi]$. The $L \times \frac{N}{2}$ discretized sampling matrix $\mathbf{B}$ can be constructed as

$$
\begin{gathered}
\mathbf{B}=\left[\mathbf{B}_{p} ; \mathbf{B}_{t} ; \mathbf{B}_{s}\right], \\
\mathbf{B}_{p}=\left[\begin{array}{c}
\mathbf{c}\left(\omega_{1}^{(p)}\right) \\
\vdots \\
\mathbf{c}\left(\omega_{L_{p}}^{(p)}\right)
\end{array}\right],\left[\omega_{1}^{(p)}, \omega_{2}^{(p)}, \cdots, \omega_{L_{p}}^{(p)}\right] \in\left[0, \omega_{0}\right), \\
\mathbf{B}_{t}=\left[\begin{array}{c}
\mathbf{c}\left(\omega_{1}^{(t)}\right) \\
\vdots \\
\mathbf{c}\left(\omega_{L_{t}}^{(t)}\right)
\end{array}\right],\left[\omega_{1}^{(t)}, \omega_{2}^{(t)}, \ldots, \omega_{L_{t}}^{(t)}\right] \in\left[\omega_{0}, \frac{\pi}{M}-\omega_{0}\right], \\
\mathbf{B}_{s}=\left[\begin{array}{c}
\mathbf{c}\left(\omega_{1}^{(s)}\right) \\
\vdots \\
\mathbf{c}\left(\omega_{L_{s}}^{(s)}\right)
\end{array}\right],\left[\omega_{1}^{(s)}, \omega_{2}^{(s)}, \cdots, \omega_{L_{s}}^{(s)}\right] \in\left(\frac{\pi}{M}-\omega_{0}, \pi\right]
\end{gathered}
$$

with $L=L_{p}+L_{t}+L_{s}$. Define $L \times 1$ vector $\mathbf{d}$ and $\mathbf{e}$ as

$$
\begin{aligned}
\mathbf{d} & =\left[\begin{array}{lllllllll}
1 & \cdots & 1 & f\left(\omega_{1}^{(t)}\right) & \cdots & f\left(\omega_{L_{t}}^{(t)}\right) & 0 & \cdots & 0
\end{array}\right]^{\mathrm{T}}, \\
\mathbf{e} & =\left[\begin{array}{llllllllll}
\delta_{p} & \cdots & \delta_{p} & \delta_{t} & \cdots & \delta_{t} & \delta_{s} & \cdots & \delta_{s}
\end{array}\right]^{\mathrm{T}} .
\end{aligned}
$$

The discretized and normalized formulation of problem Equation (17) can be expressed as: 


$$
\begin{array}{ll} 
& \min \quad\|\mathbf{h}\|_{0} \\
\text { s.t. } \quad & |\mathbf{B h}-\mathbf{d}| \leq \mathbf{e} \\
& \mathbf{c}\left(\frac{\pi}{2 M}\right) \mathbf{h}=\frac{\sqrt{2}}{2}
\end{array}
$$

Choose the initial $L \times 1$ weight vector $\mathbf{w}^{(1)}=[1, \ldots, 1]^{\mathrm{T}}$. At the $k$ iteration with $k \geq 1$, the procedure of computing the sparse FIR prototype filter proceeds as follows.

Step 1: Normalize column vectors $\mathbf{b}_{0}, \mathbf{b}_{1}, \ldots, \mathbf{b}_{\frac{N}{2}-1}$ of $\mathbf{B}$ under the weight vector $\mathbf{w}^{(k)}$ as

$$
\begin{gathered}
\mathbf{B}^{(k)}=\left[\mathbf{b}_{0}^{(k)}, \mathbf{b}_{1}^{(k)}, \ldots, \mathbf{b}_{n}^{(k)}, \ldots, \mathbf{b}_{\frac{N}{2}-1}^{(k)}\right], \\
\mathbf{b}_{n}^{(k)}=\mathbf{b}_{n} /\left[\sum_{i=0}^{L} \mathbf{w}^{(k)}(l) *\left|\mathbf{b}_{n}(l)\right|^{2}\right]^{\frac{1}{2}}, 0 \leq n \leq \frac{N}{2}-1 .
\end{gathered}
$$

Step 2: The OMP algorithm [9] is employed to solve the following problem

$$
\begin{array}{cc}
\min _{\mathbf{h}^{(k)}} & \left\|\mathbf{B}^{(k)} \mathbf{h}^{(k)}-\mathbf{d}\right\|_{2}^{2} \\
\text { s.t. } & \left\|\mathbf{h}^{(k)}\right\|_{0} \leq k .
\end{array}
$$

Let $k \times 1$ vector $\mathbf{s}^{(k)}$ denote the final solution of Equation (22), and the final $L \times 1$ residual vector is given as

$$
\mathbf{r}^{(k)}=\Phi^{(k)} \mathbf{s}^{(k)}-\mathbf{d}
$$

where $\Phi^{(k)}=\left[\mathbf{b}_{n_{1}}^{(k)}, \mathbf{b}_{n_{2}}^{(k)}, \ldots, \mathbf{b}_{n_{k}}^{(k)}\right]$ is the matrix of chosen atoms and $\Lambda^{(k)}=\left\{n_{1}, n_{2}, \ldots, n_{k}\right\}$ is the nonzero index set.

Step 3: Solve the following linear programming problem:

$$
\begin{gathered}
\min _{\mathbf{h}^{(k)}, \mu} \mu \\
\text { s.t. } \quad\left|\mathbf{B}^{(k)} \mathbf{h}^{(k)}-\mathbf{d}\right| \leq \mathbf{e}+\mu \cdot \mathbf{1}_{L \times 1}, \\
\mathbf{c}\left(\frac{\pi}{2 M}\right) \mathbf{h}^{(k)}=\frac{\sqrt{2}}{2} \\
h_{n}^{(k)}=0, \quad n \in\left\{0,1, \ldots, \frac{N}{2}-1\right\}-\Lambda^{(k)} .
\end{gathered}
$$

If $\mu \leq 0$, then the computed filter $\hat{\mathbf{h}}^{(k)}$ is the sparse FIR prototype filter of cosine-modulated filter banks which meeting our design specifications.

Step 4: Compute the new weight vector by formula from reference [10]

$$
w_{l}^{(k+1)}=\left(1+\left(\frac{\left|r_{l}^{(k)}\right|}{\max \left\{\frac{\left|\hat{\mathbf{h}}^{(k)}\right|}{100}\right\}}\right)^{2}\right)^{-\frac{1}{4}}, \quad 1 \leq l \leq L
$$

where $w_{l}^{(k+1)}$ and $r_{l}^{(k)}$ are the $i$-th entries of the new weight vector $\mathbf{w}^{(k+1)}$ and the residual vector $\mathbf{r}^{(k)}$ respectively. Replace $\mathbf{w}^{(k)}$ with $\mathbf{w}^{(k+1)}$ and repeat the procedure from Steps 1 to 4 . 


\section{Examples}

In this section, the performance of the proposed scheme is evaluated by several design examples on a Intel Core i7-6700 3.40GHz PC with MATLAB, and compares to other NPR cosine-modulated filter banks design algorithms.

Example 1. This example is concerned with the design of NPR cosine-modulated filter banks with $M=4$. The filter order of the FIR linear phase prototype filter is $N=140$. The numbers of discretized samples for pass band, transition band and stop band are $L_{p}=6, L_{t}=20$ and $L_{s}=800$, respectively. Choose $\delta_{p}=\delta_{t}=\delta_{s}=$ $1 \times 10^{-3}$. The amplitude distortion function magnitude $e_{a m}(\omega)=1-\left|A_{0}\left(e^{j \omega}\right)\right|$, the total aliasing transfer function magnitude $e_{a}(\omega)=\sqrt{\sum_{l=1}^{M-1}\left|A_{l}\left(e^{j \omega}\right)\right|^{2}}$, and the amplitude response of the sparse prototype filter derived from the proposed scheme are shown in Figure 2. The amplitude distortion $e_{a m}=\max _{\omega}\left|e_{a m}(\omega)\right|$ and total aliasing error $e_{a}=\max _{\omega}\left|e_{a}(\omega)\right|$ of the corresponding cosine-modulated filter bank are $e_{a m}^{\omega}=1.70 \times 10^{-3}$ and $e_{a}=5.06 \times 10^{-7}$, respectively. It takes 31 iterations for proposed method to converge, with the CPU time equals to 0.2716 seconds in each iteration.
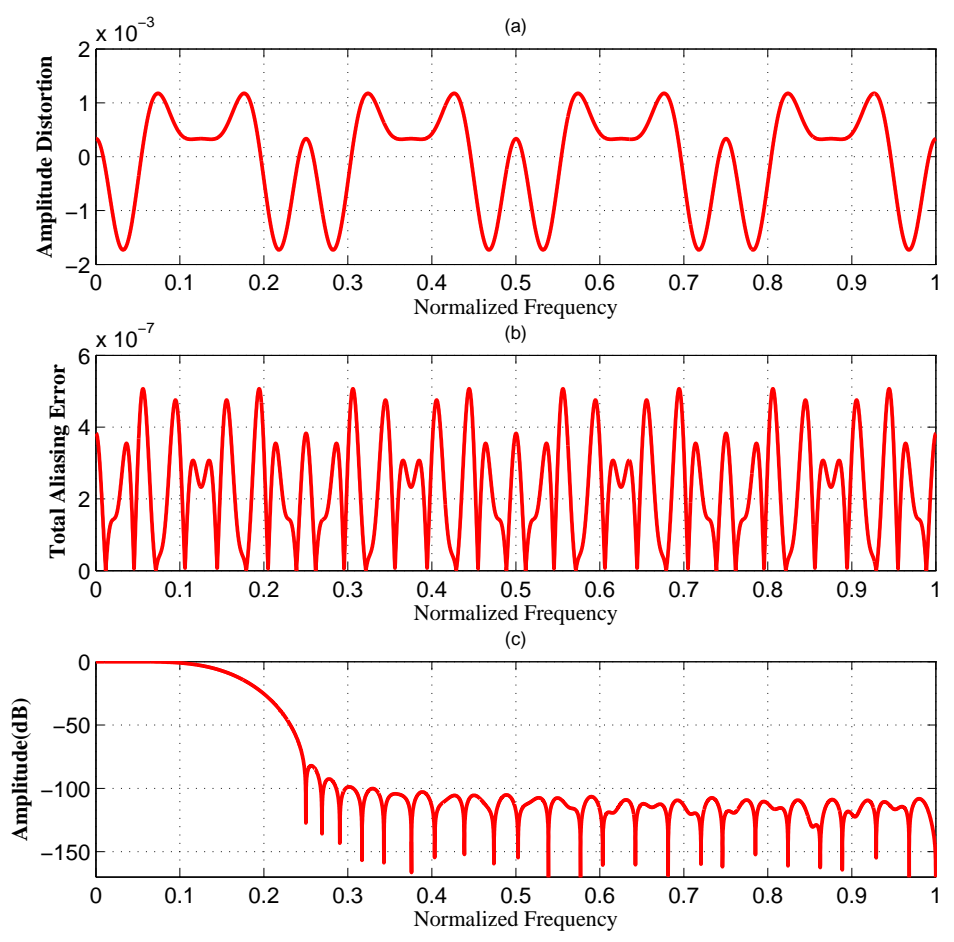

Figure 2. $M=4$ channel cosine-modulated filter banks in example 1. (a) The amplitude distortion function magnitude $e_{a m}(\omega)$; (b) The total aliasing transfer function magnitude $e_{a}(\omega)$; (c) Amplitude response of sparse prototype filter.

We compare the performance of the proposed scheme with the WCLS [6] scheme and QBPSO [7] scheme. At the similar reconstruction performances, the number of nonzero tap weights of the prototype filter yielded from the proposed method is 18 less than that of the WCLS scheme and the QBPSO scheme. In Table 1, we compare the filter order, the number of nonzero tap weights, the amplitude distortion $e_{a m}$ and the total aliasing error $e_{a}$ corresponding to the proposed scheme, the WCLS scheme and the QBPSO scheme. 
Table 1. Comparisons for Several Parameters of the Designed Filters Derived from Different Methods.

\begin{tabular}{cccccc}
\hline M & Design Method & Filter Order & Number of Nonzero Taps & Amplitude Distortion & Total Aliasing Error \\
\hline \multirow{4}{*}{4} & WCLS & 80 & 80 & $3.10 \times 10^{-3}$ & $1.06 \times 10^{-6}$ \\
& QBPSO & 80 & 80 & $7.01 \times 10^{-4}$ & $8.49 \times 10^{-8}$ \\
& Proposed Method & 140 & 62 & $1.70 \times 10^{-3}$ & $5.06 \times 10^{-7}$ \\
\hline \multirow{3}{*}{8} & WCLS & 144 & 144 & $1.80 \times 10^{-3}$ & $3.90 \times 10^{-7}$ \\
& GI & 132 & 132 & $2.14 \times 10^{-5}$ & $1.30 \times 10^{-5}$ \\
& Proposed Method & 160 & 98 & $5.20 \times 10^{-4}$ & $9.85 \times 10^{-6}$ \\
\hline \multirow{2}{*}{16} & WCLS & 224 & 224 & $1.30 \times 10^{-3}$ & $9.03 \times 10^{-7}$ \\
& QBPSO & 256 & 256 & $4.73 \times 10^{-4}$ & $2.34 \times 10^{-6}$ \\
\hline
\end{tabular}

Example 2. This example is concerned with the design of NPR cosine-modulated filter banks with $M=8$. The filter order of the FIR linear phase prototype filter is $N=160$. The numbers of discretized samples for pass band, transition band and stop band are $L_{p}=6, L_{t}=93$ and $L_{s}=91$, respectively. Choose $\delta_{p}=\delta_{t}=\delta_{s}=$ $1 \times 10^{-3}$. The amplitude distortion function magnitude $e_{a m}(\omega)$, the total aliasing transfer function magnitude $e_{a}(\omega)$, and the amplitude response of the sparse prototype filter derived from the proposed scheme are shown in Figure 3. The amplitude distortion $e_{a m}$ and total aliasing error $e_{a}$ of the corresponding cosine-modulated filter bank are $e_{a m}=5.2 \times 10^{-4}$ and $e_{a}=9.85 \times 10^{-6}$. It takes 49 iterations for proposal method to converge, with the CPU time equals to 0.0591 seconds in each iteration.

We compare the performance of the proposed scheme with the WCLS [6] scheme and GI [8] scheme. At the similar reconstruction performances, the number of nonzero tap weights of the prototype filter yielded from the proposed method is 46 less than that of the WCLS scheme, the number of nonzero tap weights of the prototype filter yielded from the proposed method is 34 less than that of the GI scheme. In Table 1, we compare the filter order, the number of nonzero tap weights, the amplitude distortion $e_{a m}$ and the total aliasing error $e_{a}$ corresponding to the proposed scheme, the WCLS scheme and the GI scheme.
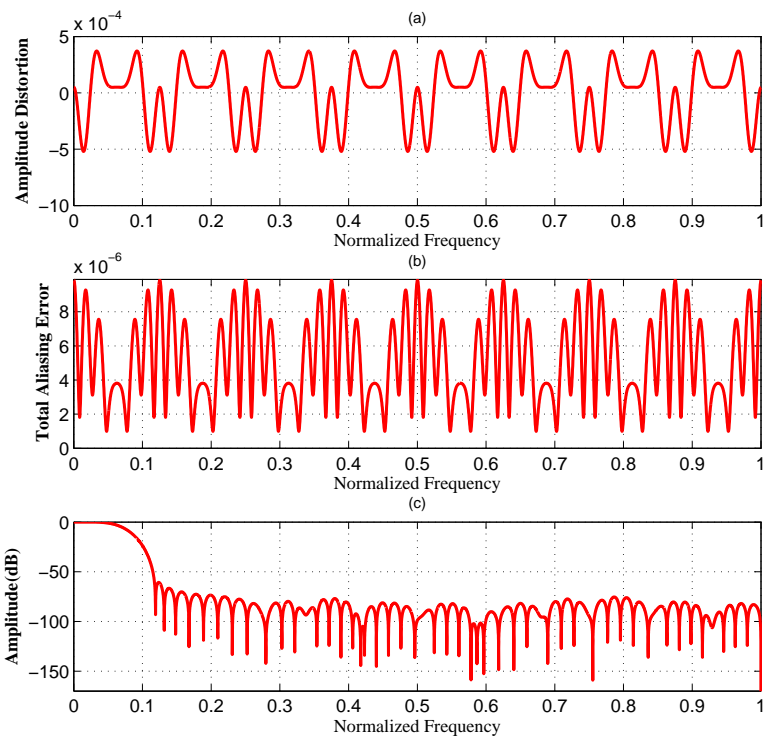

Figure 3. $M=8$ channel cosine-modulated filter banks in example 2. (a) The amplitude distortion function magnitude $e_{a m}(\omega)$; (b) The total aliasing transfer function magnitude $e_{a}(\omega)$; (c) Amplitude response of sparse prototype filter. 
Example 3. This example is concerned with the design of NPR cosine-modulated filter banks with $M=16$. The filter order of the FIR linear phase prototype filter is $N=254$. The numbers of discretized samples for pass band, transition band and stop band are $L_{p}=4, L_{t}=7$ and $L_{s}=94$, respectively. Choose $\delta_{p}=\delta_{t}=\delta_{s}=$ $1 \times 10^{-3}$. The amplitude distortion function magnitude $e_{a m}(\omega)$, the total aliasing transfer function magnitude $e_{a}(\omega)$, and the amplitude response of the sparse prototype filter derived from the proposed scheme are shown in Figure 4. The amplitude distortion $e_{a m}$ and total aliasing error $e_{a}$ of the corresponding cosine-modulated filter bank are $e_{a m}=5.21 \times 10^{-4}$ and $e_{a}=4.24 \times 10^{-6}$. It takes 83 iterations for proposed method to converge, with the CPU time equals to 0.1108 seconds in each iteration.

We compare the performance of the proposed scheme with the WCLS [6] scheme and QBPSO [7] scheme. At the similar reconstruction performances, the number of nonzero tap weights of the prototype filter yielded from the proposed method is 58 less than that of the WCLS scheme, the number of nonzero tap weights of the prototype filter yielded from the proposed method is 90 less than that of the QBPSO scheme. In Table 1, we compare the filter order, the number of nonzero tap weights, the amplitude distortion $e_{a m}$ and the total aliasing error $e_{a}$ corresponding to the proposed scheme, the WCLS scheme and the QBPSO scheme.
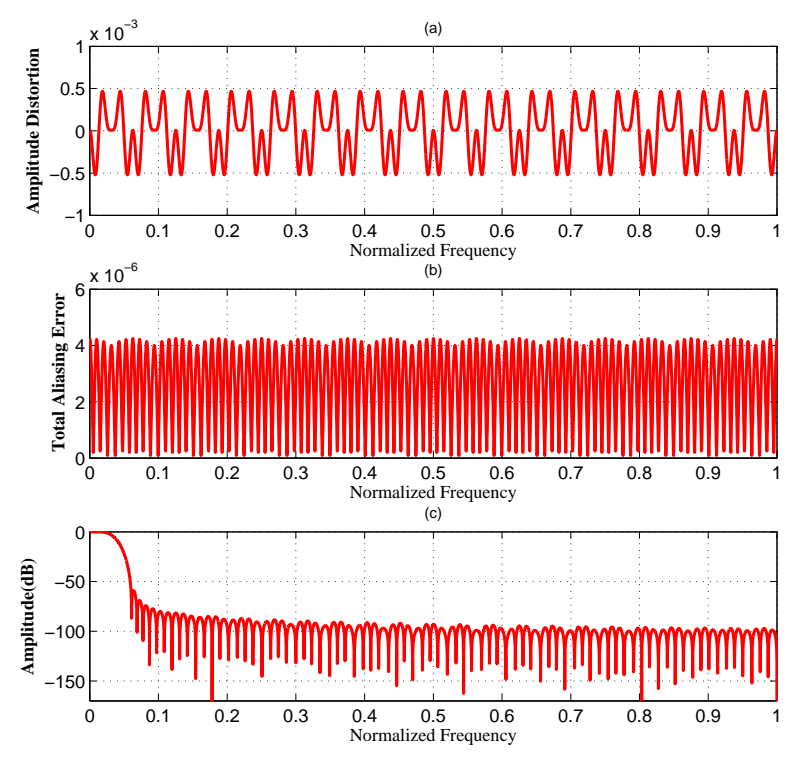

Figure 4. $M=16$ channel cosine-modulated filter banks in Example 3. (a) The amplitude distortion function magnitude $e_{a m}(\omega)$; (b) The total aliasing transfer function magnitude $e_{a}(\omega)$; (c) Amplitude response of sparse prototype filter.

\section{Conclusions}

In this paper, we present a fast design paradigm for sparse NPR cosine-modulated filter banks. Different to traditional method of designing filter banks, the proposed algorithm aims to add a sparsity into filter banks while others have not, and ensures the stop-band attenuation at least $60 \mathrm{~dB}$ while most other methods cannot guarantee those with the same performance of amplitude distortion and aliasing distortion. The desired sparse linear phase FIR prototype filter can be derived through the orthogonal matching pursuit [9] performed under the weighted $l_{2}$ norm. The simulation results demonstrate that the proposed scheme is an effective paradigm to design sparse NPR cosine-modulated filter banks.

Author Contributions: W.X. and Y.L. contributed to the overall idea and algorithm, coded the algorithm in MATLAB and contributed to the detailed writing of the manuscript. J.M., J.Z. and X.G. contributed to the revision, preparation, and publishing of the paper. All authors have read and approved the final manuscript. 
Acknowledgments: This work was supported by the Nature Science Foundation under Grant 61501324 and the Nature Science Foundation of Tianjin under Grant 14JCYBJC16100.

Conflicts of Interest: The authors declare no conflict of interest.

\section{Abbreviations}

The following abbreviations are used in this manuscript:

NPR Nearly Perfect-Reconstruction

WCLS Weighted Constrained Least Square

QBPSO Quantum-Behaved Particle Swarm Optimization

GI gradient information

OMP orthogonal matching pursuit

\section{References}

1. Hang, S.; Tian, L.; Ma, X.; Wei, Y. A reconfigurable sound wave decomposition filter bank for hearing aids based on nonlinear transformation. IEEE Tran. Biomed. Circuits Syst. 2016, 10, 487-496. [CrossRef] [PubMed]

2. Ling, B.W.; Ho, C.Y.; Teo, K.; Siu, W.; Cao, J.; Dai, Q. Optimal design of cosine modulated nonuniform linear phase FIR filter bank via both stretching and shifting frequency response of single prototype filter. IEEE Trans. Signal Proc. 2014, 62, 2517-2530. [CrossRef]

3. Kyochi, S.; Ikehara, M. A class of near shift-invariant and orientation-selective transform based on delay-less oversampled even-stacked cosine-modulated filter banks. IEICE Trans. Fundam. Electron. Commun. Comput. Sci. 2010, E93.A, 724-733. [CrossRef]

4. Zhao, N.; Pu, F.; Xu, X.; Chen, N. Cognitive wideband spectrum sensing using cosine-modulated filter banks. Int. J. Electron. 2015, 102, 1890-1901. [CrossRef]

5. Sakiyama, A.; Watanabe, K.; Tanaka, Y. Spectral graph wavelets and filter banks with low approximation error. IEEE Trans. Signal Inf. Proc. Over Netw. 2016, 2, 230-245. [CrossRef]

6. Kumar, A.; Singh, G.K.; Anand, R.S. A simple design method for the cosine-modulated filter banks using weighted constrained least square technique. J. Frankl. Inst. Geophys. 2011, 348, 606-621. j.jfranklin.2011.01.006. [CrossRef]

7. Tan, F.; Zhang, T.; Gao, C.; Huang, L. Optimal design of cosine modulated filter banks using quantum-behaved particle swarm optimization algorithm. Image Signal Proc. 2011, 5, 2280-2284. [CrossRef]

8. Zhang, Z.J. Efficient design of cosine modulated filter banks based on gradient information. IEEE Signal Proc. Lett. 2007, 14, 940-943. [CrossRef]

9. Tropp, J.A.; Gilbert, A.C. Signal recovery from random measurements via orthogonal matching pursuit. IEEE Trans. Inf. Theory 2007, 53, 4655-4666. [CrossRef]

10. Bube, K.P. Hybrid $l_{1} / l_{2}$ minimization with applications to tomography. Geophysics 1997, 62, 1183. 1.1444219. [CrossRef]

(C) 2018 by the authors. Licensee MDPI, Basel, Switzerland. This article is an open access article distributed under the terms and conditions of the Creative Commons Attribution (CC BY) license (http://creativecommons.org/licenses/by/4.0/). 